\title{
Delay-Dependent Exponential Stability for Uncertain Neutral Stochastic Systems with Mixed Delays and Markovian Jumping Parameters
}

\author{
Huabin Chen \\ Department of Mathematics, Nanchang University, Nanchang 330031, China \\ Correspondence should be addressed to Huabin Chen, chb_00721@126.com \\ Received 9 December 2011; Accepted 5 March 2012 \\ Academic Editor: Vimal Singh \\ Copyright (C) 2012 Huabin Chen. This is an open access article distributed under the Creative \\ Commons Attribution License, which permits unrestricted use, distribution, and reproduction in \\ any medium, provided the original work is properly cited. \\ This paper is mainly concerned with the globally exponential stability in mean square of uncertain \\ neutral stochastic systems with mixed delays and Markovian jumping parameters. The mixed \\ delays are comprised of the discrete interval time-varying delays and the distributed time delays. \\ Taking the stochastic perturbation and Markovian jumping parameters into account, some delay- \\ dependent sufficient conditions for the globally exponential stability in mean square of such \\ systems can be obtained by constructing an appropriate Lyapunov-Krasovskii functional, which \\ are given in the form of linear matrix inequalities (LMIs). The derived criteria are dependent on \\ the upper bound and the lower bound of the time-varying delay and the distributed delay and are \\ therefore less conservative. Two numerical examples are given to illustrate the effectiveness and \\ applicability of our obtained results.
}

\section{Introduction}

It is well known that many dynamical systems not only depend on the present and past states but also involve the derivative with delays as well as the functional of the past history. Neutral delay differential equations are often used to describe the following systems [1]:

$$
\frac{d[x(t)-D x(t-\tau)]}{d t}=f(t, x(t), x(t-\tau)) .
$$

Many authors have considered the dynamical analysis of the neutral delay differential equations (see [2-7] and references therein). For example, Chen et al. in [3] and Wu et al. in $[4,5]$ have given some LMI-based conditions ensuring the stability analysis and the 
stabilization of neutral delay systems. Taking the environmental disturbances into account, the neutral stochastic delay differential equations can be given as follows:

$$
d[x(t)-D x(t-\tau)]=f(t, x(t), x(t-\tau)) d t+g(t, x(t), x(t-\tau)) d B(t) .
$$

Some fundamental theories of neutral stochastic delay differential equations are introduced in $[1,8]$. Since they can be extensively applied into many branches for the control field, the problem about the exponential stability and the asymptotical stability of the neutral stochastic delay systems has attracted many authors' attention over the past few years, and many less conservative results of delay-dependent conditions ensuring the stabilization analysis and $H_{\infty}$ filtering design for such systems have been reported in many works, see, for example, [9-14] and references therein. The methods used include the Razumikhin-type theorems [10], the Lyapunov functional [13], the fixed point theorem [14], and the linear matrix inequality $[9,11,12]$. For example, Huang and Mao in [9] and Chen et al. in [11] have given the exponential stability criteria of neutral stochastic delay systems. Some LMI-based sufficient conditions for the mean-square exponential stability analysis of stochastic systems of neutral type have been obtained by introducing an auxiliary vector in [12]. In practice, the parameter uncertainty is considered as one of the main sources leading to undesirable behavior (e.g., instability) of dynamical systems, especially when implementing neural networks in applications. The stability analysis of the uncertain neutral stochastic system has received considerable research attention, see, for example, [15-18], and the problem of the $H_{\infty}$ filter design of the uncertain neutral stochastic delay systems has been discussed in $[19,20]$.

On the other hand, Markovian jump systems introduced by [21] are the hybrid systems with two components in the state. The first one refers to the mode that is described by a continuous-time finite-state Markovian process, and the second one refers to the state that is represented by a system of differential equations. The jump systems have the advantage of modeling the dynamic systems subject to abrupt variation in their structures, such as component failures or repairs, sudden environmental disturbance, changing subsystem interconnections, and operating in different points of a nonlinear plant [22]. The stability analysis and $H_{\infty}$ filter design of stochastic delay systems with Markovian jumping parameters and delay systems with Markovian jumping parameters have been widely studied, see, for example, [23-38]. For example, in [24], Liu et al. have discussed the exponential stability of delayed recurrent neural networks with Markovian jumping parameters; Liu et al. in [24] and Wang et al. in [25] have considered some sufficient conditions for the exponential stability of stochastic neural networks with mixed time delays and Markovian switching; Mao in [13] has also given some sufficient conditions for the exponential stability of stochastic delay interval systems with Markovian switching. More recently, He and Liu in [39] and Balasubramaniam et al. in [40] have presented some LMIbased sufficient conditions for the exponential stability of uncertain neutral systems with Markovian jumping parameters. Although Kolmanovskii et al. in [17], and Mao et al. in [18] have derived the exponential stability of the neutral stochastic delay systems with Markovian jumping parameters, some sufficient conditions obtained by using the estimate method are not easily checked. Thus, the problem of the stability analysis of the uncertain neutral stochastic systems with mixed delays and Markovian jumping parameters has not been fully investigated and there is still much room left for further consideration, which constitutes the motivation for the present research. 
In this paper, the global exponential stability of a class of the uncertain neutral stochastic systems with mixed delays and Markovian jumping parameters is discussed. The delays include the discrete and distributed time delays, and the jumping parameters are generated from a finite state Markov chain. By constructing an appropriate Lyapunov functional, some LMIs-based sufficient conditions ensuring the exponential stability in mean square of the uncertain neutral stochastic systems with mixed delays and Markovian jumping parameters are obtained by using the stochastic analysis and some bounding technique. It is worth pointing out that compared with the earlier works in $[17,18]$, the obtained results given in the form of the linear matrix inequalities (LMIs) can be easily be solved by using the standard software packages. Two illustrative examples are exploited to demonstrate the effectiveness and applicability of the obtained results.

The content of the paper is arranged as follows. In Section 2, some necessary notations, definitions, and lemmas will be introduced. In Section 3, we mainly study the exponential stability in mean square of the uncertain neutral stochastic systems with mixed delays and Markovian jumping parameters. Two illustrative numerical examples are given to show the power of our obtained results in Section 4.

Notations. Unless otherwise specified, for a real square matrix $A$, the matrix $A>0(A \geq 0$, $A<0, A \leq 0)$ means that $A$ is a positive definite (positive semidefinite, negative definite, and negative semidefinite, resp.); $\lambda_{\max }(A)$ and $\lambda_{\min }(A)$ denote the maximum and minimum eigenvalues of the square matrix $A$, respectively. Let $\left(\Omega, \Im,\left\{\Im_{t}\right\}_{t \geq 0}, P\right)$ be a probability space with a natural $\left\{\Im_{t}\right\}_{t \geq 0}$ and let $E\{\cdot\}$ stand for the mathematical expectation operator with respect to this probability measure. If $A$ is a vector or matrix, its transpose is denoted by $A^{T} .|B|=\sqrt{\operatorname{trace}\left(B^{T} B\right)}$ denotes the Euclidean norm of a vector $B$ and its induced norm of a matrix $B$. Unless explicitly stated, matrices are assumed to have real entries and compatible dimensions. Let $\tau>0$ and $C\left([-\tau, 0] ; R^{n}\right)$ be the family of all continuous $R^{n}$-valued functions $\phi$ on the interval $[-\tau, 0]$ with the norm $\|\phi\|=\sup \{|\phi(\theta)|:-\tau \leq \theta \leq 0\}$. Denote by $L_{\Im_{0}}^{2}\left([-\tau, 0] ; R^{n}\right)$ the family of all $\Im_{0}$-measurable $C\left([-\tau, 0] ; R^{n}\right)$-valued random variables $\xi=$ $\{\xi(\theta):-\tau \leq \theta \leq 0\}$ such that $\sup _{\theta \in[-\tau, 0]} E|\xi(\theta)|^{2}<+\infty . B(t)=\left[B_{1}(t), B_{2}(t), \ldots, B_{n}(t)\right]^{T}(t \geq 0)$ is an $n$-dimensional standard Brownian motion defined on the completed probability space $(\Omega, \Im, P)$.

\section{Problem Formulation}

Let $r(t)(t \geq 0)$ be a right-continuous Markov chain on the probability space taking values in a finite state space $S=\{1,2, \ldots, N\}$ with generator $\Gamma=\left\{\gamma_{i j}\right\}_{N \times N}$ given by

$$
P\{r(t+\Delta)=j \mid r(t)=i\}= \begin{cases}\gamma_{i j} \Delta+o(\Delta), & \text { if } i \neq j, \\ 1+\gamma_{i i} \Delta+o(\Delta), & \text { if } i=j,\end{cases}
$$

where $\Delta>0$. Here, $\gamma_{i j} \geq 0$ is the transition rate from $i$ to $j$ if $i \neq j$ while

$$
r_{i i}=-\sum_{j \neq i} r_{i j}
$$


Now, we assume that the Markov chain $r(\cdot)$ is independent of the Brownian motion $B(\cdot)$. It is well known that almost every sample path of $r(t)$ is a right-continuous step function with a finite number of simple jumps in any finite subinterval of $R^{+}$.

In this paper, we will consider the following uncertain neutral stochastic systems with mixed delays and Markovian switching:

$$
\begin{aligned}
d[x(t)-D(r(t)) x(t-d(t))]= & {\left[A_{1}(r(t), t) x(t)+A_{2}(r(t), t) f(x(t))+A_{3}(r(t), t) f(x(t-d(t)))\right.} \\
& \left.+A_{4}(r(t), t) \int_{t-\sigma(t)}^{t} f(x(s)) d s\right] d t \\
& +\sigma\left(t, x(t), x(t-d(t)), \int_{t-\sigma(t)}^{t} x(s) d s, r(t)\right) d B(t), \quad t \geq 0,
\end{aligned}
$$

with the initial value $x_{0}=\varphi \in L_{\Im_{0}}^{2}\left([-\tau, 0], R^{n}\right)\left(\tau=\max \left\{d_{2}, \sigma\right\}\right)$, where $x(t) \in R^{n}$ is the system state vector associated with the neurons and $d(t)$ and $\sigma(t)$ are the time-varying delays. Here, we assume that the Markov chain $r(\cdot)$ is independent of the Brownian motion $B(t)(t \geq 0)$. $f: R^{n} \rightarrow R^{n}$ is neuron activation function, and the noise perturbation $\sigma: R^{+} \times R^{n} \times R^{n} \times R^{n} \times$ $S \rightarrow R^{n \times n}$ is the noise intensity matrix. When $r(t)=i(i \in S), D(r(t)), A_{1}(r(t), t), A_{2}(r(t), t)$, $A_{3}(r(t), t)$, and $A_{4}(r(t), t)$ are, respectively, denoted as $D_{i}, A_{1 i}(t), A_{2 i}(t), A_{3 i}(t)$, and $A_{4 i}(t)$, and $D_{i}(i \in S)$ are known matrices with $\left|D_{i}\right|<1(i \in S) . A_{1 i}(t), A_{2 i}(t), A_{3 i}(t)$, and $A_{4 i}(t)$ are matrix functions with time-varying uncertainties, that is,

$$
\begin{aligned}
& A_{1 i}(t)=A_{1 i}+\Delta A_{1 i}(t), \\
& A_{2 i}(t)=A_{2 i}+\Delta A_{2 i}(t), \\
& A_{3 i}(t)=A_{3 i}+\Delta A_{3 i}(t), \\
& A_{4 i}(t)=A_{4 i}+\Delta A_{4 i}(t),
\end{aligned}
$$

where $A_{1 i}, A_{2 i}, A_{3 i}$, and $A_{4 i}(i \in S)$ are known real constant matrices and $\Delta A_{1 i}(t), \Delta A_{2 i}(t)$, $\Delta A_{3 i}(t)$, and $\Delta A_{4 i}(t)(i \in S)$ are unknown matrices representing time-varying parameter uncertainties in system model. We assume that the uncertainties are norm-bounded and can be described as

$$
\left[\Delta A_{1 i}(t) \quad \Delta A_{2 i}(t) \quad \Delta A_{3 i}(t) \quad \Delta A_{4 i}(t)\right]=M_{i} F_{i}(t)\left[N_{1 i} N_{2 i} N_{3 i} N_{4 i}\right], \quad F_{i}^{T}(t) F_{i}(t) \leq I, \quad i \in S,
$$

where $M_{i}, N_{1 i}, N_{2 i}, N_{3 i}$, and $N_{4 i}(i \in S)$ are known real matrices and $F_{i}(t)(i \in S)$ is unknown real and possibly time-varying matrix for any given $t$. It is assumed that the elements of 
$F_{i}(t)(i \in S)$ are Lebesgue measurable. When $F_{i}(t)=0(i \in S)$, systems (2.3) have the following nominal case:

$$
\begin{aligned}
d\left[x(t)-D_{i} x(t-d(t))\right]= & {\left[A_{1 i} x(t)+A_{2 i} f(x(t))+A_{3 i} f(x(t-d(t)))+A_{4 i} \int_{t-\sigma(t)}^{t} f(x(s)) d s\right] d t } \\
& +\sigma\left(t, x(t), x(t-d(t)), \int_{t-\sigma(t)}^{t} x(s) d s, i\right) d B(t), \quad t \geq 0 .
\end{aligned}
$$

In order to obtain our results, we need some assumptions as follows.

Assumption 2.1. The neuron activation functions $f(\cdot)$ in (2.3) (or (2.6)) are bounded and satisfy the following Lipschitz condition:

$$
|f(x)-f(y)| \leq|L(x-y)|, \quad \forall x, y \in R^{n},
$$

where $L \in R^{n \times n}$ is known constant matrix and $f(0)=0$.

Assumption 2.2. The noise perturbation $\sigma$ satisfies the following condition:

$$
\begin{aligned}
& \operatorname{trace}\left[\sigma^{T}\left(t, x(t), x(t-d(t)), \int_{t-\sigma(t)}^{t} x(s) d s, i\right) \sigma\left(t, x(t), x(t-d(t)), \int_{t-\sigma(t)}^{t} x(s) d s, i\right)\right] \\
& \quad \leq x^{T}(t) R_{1 i}^{T} R_{1 i} x(t)+x^{T}(t-d(t)) R_{2 i}^{T} R_{2 i} x(t-d(t))+\left[\int_{t-\sigma(t)}^{t} x(s) d s\right]^{T} R_{3 i}^{T} R_{3 i}\left[\int_{t-\sigma(t)}^{t} x(s) d s\right],
\end{aligned}
$$

where $R_{1 i}, R_{2 i}$, and $R_{3 i}$ are known constant matrices with appropriate dimensions and $\sigma(t, 0$, $0,0, i)=0(i \in S)$.

Remark 2.3. Under Assumptions 2.1 and 2.2, it is easily shown that the system (2.3) with uncertainties (2.4) admits a unique trivial solution when the initial data $\xi=0$. The readers can refer to [41].

Assumption 2.4. $d(t)$ and $\sigma(t)$ are two time-varying continuous functions that satisfy

$$
0 \leq d_{1} \leq d(t) \leq d_{2}, \quad \dot{d}(t) \leq \mu<1, \quad 0 \leq \sigma(t) \leq \sigma, \quad \dot{\sigma}(t) \leq v<1,
$$

where $d_{1}$ and $d_{2}$ are the lower and upper bounds of the time delay $d(t)$, respectively.

We present the definitions and three useful lemmas as follows. 
Definition 2.5. The neutral stochastic systems with mixed delays and Markovian jump parameters (2.6) is said to be exponentially stable in mean square if there exist a pair of positive scalar $\alpha>0$ and $l>0$ such that every solution $x(t, \xi, i)$ of systems (2.6) satisfies

$$
E|x(t, \xi, i)|^{2} \leq l \sup _{s \in[-\tau, 0]} E|\varphi(s)|^{2} e^{-\alpha t}, \quad \forall t \geq 0
$$

for any $\varphi \in L_{\Im_{0}}^{2}\left([-\tau, 0], R^{n}\right)$.

Definition 2.6. The uncertain neutral stochastic systems with mixed delays and Markovian jump parameters (2.3) are said to be exponentially stable in mean square if (2.10) holds for all admissible uncertainties (2.5).

Lemma 2.7 (see [8]). For any vectors $a, b \in R^{n}$, the inequality

$$
\pm 2 a^{T} b \leq a^{T} X a+b^{T} X^{-1} b
$$

holds, in which $X$ is any $n \times n$ matrix with $X>0$.

Lemma 2.8 (see [8]). Let $X \in R^{n \times n}$; then

$$
\lambda_{\min }(X) a^{T} b \leq a^{T} X b \leq \lambda_{\max }(X) a^{T} b
$$

for any $a \in R^{n}$ if $X$ is a symmetric matrix.

Lemma 2.9 (see [42] Schur complement). For a given matrix

$$
S=\left(\begin{array}{ll}
S_{11} & S_{12} \\
S_{12}^{T} & S_{22}
\end{array}\right)
$$

with $S_{11}=S_{11}^{T}, S_{22}=S_{22}^{T}$, the following conditions are equivalent:

(1) $S<0$,

(2) $S_{22}<0, S_{11}-S_{12} S_{22}^{-1} S_{12}^{T}<0$,

(3) $S_{11}<0, S_{22}-S_{12}^{T} S_{11}^{-1} S_{12}<0$.

Lemma 2.10 (see [42]). Let $U, V, W$, and $M$ be real matrices of appropriate dimensions with $M$ satisfying $M=M^{T}$; then

$$
M+U V W+W^{T} V^{T} U^{T}<0, \quad \forall V^{T} V \leq I,
$$

if and only if there exist a scalar $\varepsilon>0$ such that

$$
M+\varepsilon^{-1} U U^{T}+\varepsilon W^{T} W<0 .
$$


Lemma 2.11 (see [43]). For any positive symmetric constant matrix $M \in R^{n \times n}$ and a scalar $\gamma>0$, a vector function $\omega:[0, \gamma] \rightarrow R^{n}$ such that the integrations concerned are well defined, and then the following inequality holds:

$$
\left[\int_{0}^{\gamma} \omega(s) d s\right]^{T} M\left[\int_{0}^{\gamma} \omega(s) d s\right] \leq \gamma \int_{0}^{\gamma} \omega^{T}(s) M \omega(s) d s
$$

\section{Main Results}

Theorem 3.1. Suppose that Assumptions 2.1-2.4 hold and for any given positive scalar $\kappa \in(0,1)$, the neutral stochastic systems with mixed delays and Markovian switching (2.6) are exponentially stable in mean square if there exist $\lambda_{i}>0(i \in S)$ and some positive definite matrices $P_{i}>0(i \in S)$ and $Q_{l}>0(l=1,2, \ldots, 10)$ such that the following linear matrix inequalities (LMIs) are satisfied: for $i \in S$,

$$
\Omega_{i}=\left[\begin{array}{ccccccccc}
\Omega_{i 11} & \Omega_{i 12} & 0 & P_{i} A_{2 i} & P_{i} A_{3 i} & P_{i} A_{4 i} & 0 & 0 & 0 \\
* & \Omega_{i 22} & 0 & 0 & 0 & 0 & \Omega_{i 27} & \Omega_{i 28} & \Omega_{i 29} \\
* & * & \Omega_{i 33} & 0 & 0 & 0 & 0 & 0 & 0 \\
* & * & * & -Q_{5} & 0 & 0 & 0 & 0 & 0 \\
* & * & * & * & -Q_{6} & 0 & 0 & 0 & 0 \\
* & * & * & * & * & -Q_{7} & 0 & 0 & 0 \\
* & * & * & * & * & * & -Q_{8} & 0 & 0 \\
* & * & * & * & * & * & * & -Q_{9} & 0 \\
* & * & * & * & * & * & * & * & -Q_{10}
\end{array}\right]<0,
$$

where * denotes the entries that are readily inferred by symmetry of a symmetric matrix and

$$
\begin{gathered}
\Omega_{i 11}=P_{i} A_{1 i}+A_{1 i}^{T} P_{i}+\sum_{j=1}^{N} \gamma_{i j} P_{j}+\lambda_{i} R_{1 i}^{T} R_{1 i}+L^{T}\left(Q_{5}+Q_{8}\right) L+Q_{1}+d_{2} Q_{2}+\frac{1}{2}\left(d_{2}^{2}-d_{1}^{2}\right) Q_{3}+\sigma^{2} Q_{4}, \\
\Omega_{i 12}=A_{1 i}^{T} P_{i} D_{i}-\sum_{j=1}^{N} \gamma_{i j} P_{j} D_{i}, \\
\Omega_{i 22}=L^{T}\left(Q_{6}+Q_{9}\right) L+\sum_{j=1}^{N} \gamma_{i j} D_{i}^{T} P_{j} D_{i}+\lambda_{i} R_{2 i}^{T} R_{2 i}-(1-u) Q_{1}, \\
\Omega_{i 33}=L^{T}\left(Q_{7}+Q_{10}\right) L+\lambda_{i} R_{3 i}^{T} R_{3 i}-\kappa(1-v) Q_{5}, \\
\Omega_{i 27}=D_{i}^{T} P_{i} A_{2 i}, \quad \Omega_{i 28}=D_{i}^{T} P_{i} A_{3 i}, \quad \Omega_{i 29}=D_{i}^{T} P_{i} A_{4 i} .
\end{gathered}
$$


Proof. Denote by $C^{2,1}\left(R^{+} \times R^{n} \times S ; R^{n}\right)$ the family of all nonnegative functions $V(t, x, i)$ on $R^{+} \times$ $R^{n} \times S$ that are once differentiable with respect to the first variable $t$ and twice differentiable with respect to the second variable $x$. To obtain the stability conditions, we consider the following Lyapunov functional:

$$
V(t, x(t), i)=V_{1}(t, x(t), i)+V_{2}(t, x(t), i)+V_{3}(t, x(t), i)
$$

where

$$
\begin{gathered}
V_{1}(t, x(t), i)=\left[x(t)-D_{i} x(t-d(t))\right]^{T} P_{i}\left[x(t)-D_{i} x(t-d(t))\right], \\
V_{2}(t, x(t), i)=\int_{t-d(t)}^{t} x^{T}(s) Q_{1} x(s) d s+\int_{t-d(t)}^{t} \int_{s}^{t} x^{T}(\theta) Q_{2} x(\theta) d \theta d s, \\
V_{3}(t, x(t), i)=\int_{-d_{2}}^{-d_{1}} \int_{t+s}^{t} \int_{\theta}^{t} x^{T}(u) Q_{3} x(u) d u d \theta d s+\sigma \int_{-\sigma(t)}^{0} \int_{t+s}^{t} x^{T}(\theta) Q_{4} x(\theta) d \theta d s .
\end{gathered}
$$

The weak infinitesimal operator $\mathfrak{L} V$ [17] along (2.6) from $R^{+} \times R^{n} \times S$ to $R$ is given by

$$
\mathfrak{L} V(t, x(t), i)=\mathfrak{L} V_{1}(t, x(t), i)+\mathfrak{L} V_{2}(t, x(t), i)+\mathfrak{L} V_{3}(t, x(t), i),
$$

where

$$
\begin{aligned}
\mathfrak{L} V_{1}(t, x(t), i) & =x^{T}(t)\left[P_{i} A_{1 i}+A_{1 i}^{T} P_{i}+\sum_{j=1}^{N} \gamma_{i j} P_{j}\right] x(t)+2 x^{T}(t) P_{i} A_{2 i} f(x(t)) \\
& +2 x^{T}(t) P_{i} A_{3 i} f(x(t-d(t)))+2 x^{T}(t) P_{i} A_{4 i} \int_{t-\sigma(t)}^{t} f(x(s)) d s \\
& +2 x^{T}(t-d(t)) D_{i}^{T} P_{i} A_{1 i} x(t)-2 x^{T}(t-d(t)) D_{i}^{T} P_{i} A_{2 i} f(x(t)) \\
& +2 x^{T}(t-d(t)) D_{i}^{T} P_{i} A_{3 i} f(x(t-d(t)))-2 x^{T}(t-d(t)) D_{i}^{T} P_{i} A_{4 i} \int_{t-\sigma(t)}^{t} f(x(s)) d s \\
& +x^{T}(t)\left[-\sum_{j=1}^{N} \gamma_{i j} P_{j} D_{i}\right] x(t-d(t))+x^{T}(t-d(t))\left[-\sum_{j=1}^{N} \gamma_{i j} D_{i}^{T} P_{i}\right] x(t) \\
& +x^{T}(t-d(t))\left[\sum_{j=1}^{N} D_{i}^{T} P_{j} D_{i}\right] x(t-d(t)) \\
& +\operatorname{trace}\left[\sigma^{T}\left(t, x(t), x(t-d(t)), \int_{t-\sigma(t)}^{t} x(s) d s\right) V_{1 x x} \sigma\left(t, x(t), x(t-d(t)), \int_{t-\sigma(t)}^{t} x(s) d s\right)\right] .
\end{aligned}
$$


By Lemma 2.7, we have

$$
\begin{aligned}
x^{T}(t) P_{i} A_{2 i} f(x(t), i) \leq & x^{T}(t) P_{i} A_{2 i} Q_{5}^{-1} A_{2 i}^{T} P_{i} x(t)+f^{T}(x(t)) Q_{5} f(x(t)) \\
\leq & x^{T}(t) P_{i} A_{2 i} Q_{5}^{-1} A_{2 i}^{T} P_{i} x(t)+x^{T}(t) L^{T} Q_{5} L x(t), \\
2 x^{T}(t) P_{i} A_{3 i} f(x(t-d(t)), i) \leq & x^{T}(t) P_{i} A_{3 i} Q_{6}^{-1} A_{3 i}^{T} P_{i} x(t) \\
& +f^{T}(x(t-d(t)), i) Q_{6} f(x(t-d(t)), i) \\
\leq & x^{T}(t) P_{i} A_{3 i} Q_{6}^{-1} A_{3 i}^{T} P_{i} x(t) \\
& +x^{T}(t-d(t)) L^{T} Q_{6} L x(t-d(t)), \\
-2 x^{T}(t-d(t)) D_{i}^{T} P_{i} A_{2 i} f(x(t), i) \leq & x^{T}(t-d(t)) D_{i}^{T} P_{i} A_{2 i} Q_{8}^{-1} A_{2 i}^{T} P_{i} D_{i} x(t-d(t)) \\
& +f^{T}(x(t)) Q_{8} f(x(t)) \\
\leq & x^{T}(t-d(t)) D_{i}^{T} P_{i} A_{2 i} Q_{8}^{-1} A_{2 i}^{T} P_{i} D_{i} x(t-d(t)) \\
& +x^{T}(t) L^{T} Q_{8} L x(t), \\
-2 x^{T}(t-d(t)) D_{i}^{T} P_{i} A_{3 i} f(x(t-d(t)), i) \leq & x^{T}(t-d(t)) D_{i}^{T} P_{i} A_{3 i} Q_{9}^{-1} A_{3 i}^{T} P_{i} D_{i} x(t-d(t)) \\
& +f^{T}(x(t-d(t))) Q_{9} f(x(t-d(t))) \\
\leq & x^{T}(t-d(t)) D_{i}^{T} P_{i} A_{3 i} Q_{9}^{-1} A_{3 i}^{T} P_{i} D_{i} x(t-d(t)) \\
& +x^{T}(t-d(t)) L^{T} Q_{9} L x(t-d(t)) .
\end{aligned}
$$

From Lemmas 2.7 and 2.11, it implies that

$$
\begin{aligned}
2 x^{T}(t) P_{i} A_{4 i} \int_{t-\sigma(t)}^{t} f(x(s)) d s \leq & x^{T}(t) P_{i} A_{4 i} Q_{7}^{-1} A_{4 i}^{T} P_{i} x(t) \\
+ & {\left[\int_{t-\sigma(t)}^{t} f(x(s)) d s\right]^{T} Q_{7}\left[\int_{t-\sigma(t)}^{t} f(x(s)) d s\right] } \\
\leq & x^{T}(t) P_{i} A_{4 i} Q_{7}^{-1} A_{4 i}^{T} P_{i} x(t) \\
+ & {\left[\int_{t-\sigma(t)}^{t} x(s) d s\right]^{T} L^{T} Q_{7} L\left[\int_{t-\sigma(t)}^{t} x(s) d s\right] } \\
-2 x^{T}(t-d(t)) D_{i}^{T} P_{i} A_{4 i} \int_{t-\sigma(t)}^{t} f(x(s)) d s \leq & x^{T}(t-d(t)) D_{i}^{T} P_{i} A_{4 i} Q_{10}^{-1} A_{4 i}^{T} P_{i} D_{i} x(t-d(t)) \\
+ & {\left[\int_{t-\sigma(t)}^{t} f(x(s)) d s\right]^{T} Q_{10}\left[\int_{t-\sigma(t)}^{t} f(x(s)) d s\right] } \\
\leq & x^{T}(t-d(t)) P_{i} A_{4 i} Q_{10}^{-1} A_{4 i}^{T} P_{i} x(t-d(t)) \\
& +\left[\int_{t-\sigma(t)}^{t} x(s) d s\right]^{T} L^{T} Q_{10} L\left[\int_{t-\sigma(t)}^{t} x(s) d s\right] .
\end{aligned}
$$


Substituting (3.8)-(3.13) into (3.7) and from Assumption 2.2, it follows that

$$
\begin{aligned}
& \mathfrak{L} V_{1}(t, x(t), i) \\
& \leq x^{T}(t)\left[P_{i} A_{1 i}+A_{1 i}^{T} P_{i}+\sum_{j=1}^{N} \gamma_{i j} P_{j}+\lambda_{i} R_{1 i}^{T} R_{1 i}+L^{T}\left(Q_{5}+Q_{8}\right) L\right. \\
& \left.+P_{i} A_{2 i} Q_{5}^{-1} A_{2 i}^{T} P_{i}+P_{i} A_{3 i}(t) Q_{6}^{-1} A_{3 i}^{-T} P_{i}+P_{i} A_{4 i} Q_{7}^{-1} A_{4 i}^{T} P_{i}\right] x(t) \\
& +x^{T}(t)\left[A_{1 i}^{T} P_{i} D_{i}-\sum_{j=1}^{N} \gamma_{i j} P_{j} D_{i}\right] x(t-d(t))+x^{T}(t-d(t)) \\
& \times\left[D_{i}^{T} P_{i} A_{1 i}-\sum_{j=1}^{N} \gamma_{i j} D_{i}^{T} P_{j}\right] x(t)+x(t-d(t)) \\
& \times\left[D_{i}^{T} P_{i} A_{2 i} Q_{8}^{-1} A_{2 i}^{T} P_{i} D_{i}+D_{i}^{T} P_{i} A_{3 i} Q_{9}^{-1} A_{3 i}^{T} P_{i} D_{i}\right. \\
& \left.+D_{i}^{T} P_{i} A_{4 i} Q_{10}^{-1} A_{4 i}^{T} P_{i} D_{i}+L^{T}\left(Q_{6}+Q_{9}\right) L+\sum_{j=1}^{N} \gamma_{i j} D_{i}^{T} P_{j} D_{i}+\lambda_{i} R_{2 i}^{T} R_{2 i}\right] x(t-d(t)) \\
& +\left[\int_{t-\sigma(t)}^{t} x(s) d s\right]^{T}\left[L^{T}\left(Q_{7}+Q_{10}\right) L+\lambda_{i} R_{3 i}^{T} R_{3 i}\right]\left[\int_{t-\sigma(t)}^{t} x(s) d s\right] .
\end{aligned}
$$

On the other hand, we can obtain

$$
\begin{aligned}
\mathfrak{L} V_{2}(t, x(t), i) \leq & x^{T}\left[Q_{1}+d_{2} Q_{2}\right] x(t)-(1-\mu) x^{T}(t-d(t)) Q_{1} x(t-d(t)) \\
& -(1-\mu) \int_{t-d(t)}^{t} x^{T}(s) Q_{2} x(s) d s \\
\mathfrak{L} V_{3}(t, x(t), i) \leq & x^{T}(t)\left[\frac{1}{2}\left(d_{2}^{2}-d_{1}^{2}\right) Q_{3}+\sigma^{2} Q_{4}\right] x(t)-\int_{-d_{2}}^{-d_{1}} \int_{t+s}^{t} x^{T}(\theta) Q_{3} x(\theta) d \theta d s \\
& -\sigma(1-v) \int_{t-\sigma(t)}^{t} x^{T}(s) Q_{5} x(s) d s \\
\leq & x^{T}(t)\left[\frac{1}{2}\left(d_{2}^{2}-d_{1}^{2}\right) Q_{3}+\sigma^{2} Q_{4}\right] x(t)-\int_{-d_{2}}^{-d_{1}} \int_{t+s}^{t} x^{T}(\theta) Q_{3} x(\theta) d \theta d s \\
& -\sigma(1-\kappa)(1-v) \int_{t-\sigma(t)}^{t} x^{T}(s) Q_{5} x(s) d s \\
& -\kappa(1-v)\left[\int_{t-\sigma(t)}^{t} x(s) d s\right]^{T} Q_{5}\left[\int_{t-\sigma(t)}^{t} x(s) d s\right] .
\end{aligned}
$$


Substituting (3.14)-(3.16) into (3.6), we have

$$
\begin{aligned}
& \mathfrak{L} V(t, x(t), i) \\
& \leq x^{T}(t)\left[P_{i} A_{1 i}+A_{1 i}^{T} P_{i}+\sum_{j=1}^{N} \gamma_{i j} P_{j}+\lambda_{i} R_{1 i}^{T} R_{1 i}+L^{T}\left(Q_{5}+Q_{8}\right) L+P_{i} A_{2 i} Q_{5}^{-1} A_{2 i}^{T} P_{i}\right. \\
& \left.+P_{i} A_{3 i}(t) Q_{6}^{-1} A_{3 i}^{T} P_{i}+P_{i} A_{4 i} Q_{7}^{-1} A_{4 i}^{T} P_{i}+Q_{1}+d_{2} Q_{2}+\frac{1}{2}\left(d_{2}^{2}-d_{1}^{2}\right) Q_{3}+\sigma^{2} Q_{4}\right] x(t) \\
& +x^{T}(t)\left[A_{1 i}^{T} P_{i} D_{i}-\sum_{j=1}^{N} \gamma_{i j} P_{j} D_{i}\right] x(t-d(t))+x(t-d(t))\left[D_{i}^{T} P_{i} A_{1 i}^{T}-\sum_{j=1}^{N} \gamma_{i j} D_{i}^{\mathrm{T}} P_{j}\right] x(t) \\
& +x^{T}(t-d(t))\left[L^{T}\left(Q_{6}+Q_{9}\right) L+D_{i}^{T} P_{i} A_{2 i} Q_{8}^{-1} A_{2 i}^{T} P_{i} D_{i}+D_{i}^{T} P_{i} A_{3 i} Q_{9}^{-1} A_{3 i}^{T} P_{i} D_{i}\right. \\
& \left.+D_{i}^{T} P_{i} A_{4 i} Q_{10}^{-1} A_{4 i}^{T} P_{i} D_{i}+\lambda_{i} R_{2 i}^{T} R_{2 i}+\sum_{j=1}^{N} \gamma_{i j} D_{i}^{T} P_{j} D_{i}-(1-\mu) Q_{1}\right] x(t-d(t)) \\
& +\left[\int_{t-\sigma(t)}^{t} x(s) d s\right]^{T}\left[L^{T}\left(Q_{7}+Q_{10}\right) L+\lambda_{i} R_{3 i}^{T} R_{3 i}-\kappa(1-v) Q_{4}\right]\left[\int_{t-\sigma(t)}^{t} x(s) d s\right] \\
& -(1-\mu) \int_{t-d(t)}^{t} x^{T}(s) Q_{2} x(s) d s-\int_{-d_{2}}^{-d_{1}} \int_{t+s}^{t} x^{T}(\theta) Q_{3} x(\theta) d \theta d s \\
& -\sigma(1-\kappa)(1-v) \int_{t-\sigma(t)}^{t} x^{T}(s) Q_{5} x(s) d s \\
& \leq \xi^{T}(t) \Pi_{i} \xi(t)-(1-\mu) \int_{t-d(t)}^{t} x^{T}(s) Q_{2} x(s) d s-\int_{-d_{2}}^{-d_{1}} \int_{t+s}^{t} x^{T}(\theta) Q_{3} x(\theta) d \theta d s \\
& -\sigma(1-\kappa)(1-v) \int_{t-\sigma(t)}^{t} x^{T}(s) Q_{4} x(s) d s,
\end{aligned}
$$

where $\xi(t)=\left[x^{T}(t) x^{T}(t-d(t)) \int_{t-\sigma(t)}^{t} x^{T}(s) d s\right]^{T}$ and, for $i \in S$,

$$
\begin{aligned}
\Pi_{i}= & {\left[\begin{array}{ccc}
\Pi_{11} & \Pi_{12} & 0 \\
* & \Pi_{22} & 0 \\
* & * & \Pi_{33}
\end{array}\right], } \\
\Pi_{11}= & P_{i} A_{1 i}+A_{1 i}^{T} P_{i}+\sum_{j=1}^{N} \gamma_{i j} P_{j}+\lambda_{i} R_{1 i}^{T} R_{1 i}+L^{T}\left(Q_{5}+Q_{8}\right) L+P_{i} A_{2 i} Q_{5}^{-1} A_{2 i}^{T} P_{i} \\
& +P_{i} A_{3 i}(t) Q_{6}^{-1} A_{3 i}^{T} P_{i}+P_{i} A_{4 i} Q_{7}^{-1} A_{4 i}^{T} P_{i}+Q_{1}+d_{2} Q_{2}+\frac{1}{2}\left(d_{2}^{2}-d_{1}^{2}\right) Q_{3}+\sigma^{2} Q_{4},
\end{aligned}
$$




$$
\begin{aligned}
\Pi_{12}= & A_{1 i}^{T} P_{i} D_{i}-\sum_{j=1}^{N} P_{j} D_{i} \\
\Pi_{22}= & L^{T}\left(Q_{6}+Q_{9}\right) L+D_{i}^{T} P_{i} A_{2 i} Q_{8}^{-1} A_{2 i}^{T} P_{i} D_{i}+D_{i}^{T} P_{i} A_{3 i} Q_{9}^{-1} A_{3 i}^{T} P_{i} D_{i} \\
& +D_{i}^{T} P_{\mathrm{i}} A_{4 i} Q_{10}^{-1} A_{4 i}^{T} P_{i} D_{i}+\lambda_{i} R_{2 i}^{T} R_{2 i}+\sum_{j=1}^{N} \gamma_{i j} D_{i}^{T} P_{j} D_{i}-(1-\mu) Q_{1}, \\
\Pi_{33}= & L^{T}\left(Q_{7}+Q_{10}\right) L+\lambda_{i} R_{3 i}^{T} R_{3 i}-\kappa(1-v) Q_{4} .
\end{aligned}
$$

In view of (3.2), we have $\Pi_{i}<0$, for $i \in S$. By the Lyapunov functional $V(t, x(t), i)$,

$$
\begin{aligned}
V(t, x(t), i)= & {\left[x(t)-D_{i} x(t-d(t))\right]^{T} P_{i}\left[x(t)-D_{i} x(t-d(t))\right]+\int_{t-d(t)}^{t} x^{T}(s) Q_{1} x(s) d s } \\
& +\int_{t-d(t)}^{t} \int_{s}^{t} x^{T}(\theta) Q_{2} x(\theta) d \theta d s+\int_{-d_{2}}^{-d_{1}} \int_{t+s}^{t} \int_{\theta}^{t} x^{T}(u) Q_{3} x(u) d u d \theta d s \\
& +\sigma \int_{-\sigma(t)}^{0} \int_{t+s}^{t} x^{T}(\theta) Q_{4} x(\theta) d \theta d s \\
\leq & \xi^{T}(t) \Pi_{i}^{\prime} \xi(t)+\int_{t-d(t)}^{t} x^{T}(s)\left[Q_{1}+d_{2} Q_{2}\right] x(s) d s+\sigma^{2} \int_{t-\sigma(t)}^{t} x^{T}(s) Q_{4} x(s) d s \\
& +\int_{-d_{2}}^{-d_{1}} \int_{t+s}^{t} x^{T}(\theta) d_{2} Q_{3} x(\theta) d \theta d s,
\end{aligned}
$$

where

$$
\Pi_{i}^{\prime}=\left[\begin{array}{ccc}
P_{i} & -P_{i} D_{i} & 0 \\
-D_{i}^{T} P_{i} & D_{i}^{T} P_{i} D_{i} & 0 \\
0 & 0 & 0
\end{array}\right] .
$$

Letting $\alpha>0$, for system (2.6), we can define another operator $\mathfrak{L}\left[e^{\alpha t} V(t, x(t), i)\right]: R^{+} \times$ $R^{n} \times S \rightarrow R$ as follows:

$$
\begin{aligned}
\mathfrak{L}\left[e^{\alpha t} V(t, x(t), i)\right]= & e^{\alpha t}[\alpha V(t, x(t), i)+\mathfrak{L} V(t, x(t), i)] \\
\leq & e^{\alpha t} \xi^{T}(t)\left[\alpha \Pi_{i}^{\prime}+\Pi_{i}\right] \xi(t)+\int_{t-d(t)}^{t} x^{T}(s)\left[\alpha\left(Q_{1}+d_{2} Q_{2}\right)-(1-\mu) Q_{2}\right] x(s) d s \\
& +\int_{-d_{2}}^{-d_{1}} \int_{t+s}^{t} x^{T}(\theta)\left[\alpha d_{2}-1\right] Q_{3} x(\theta) d \theta d s \\
& +\int_{t-\sigma(t)}^{t} x^{T}(s)\left[\alpha \sigma^{2}-\sigma(1-\kappa)(1-v)\right] Q_{4} d s .
\end{aligned}
$$


Discrete Dynamics in Nature and Society

Now, we can choose $\alpha>0$ sufficiently small such that

$$
\begin{gathered}
\alpha \lambda_{\max }\left(\Pi_{i}^{\prime}\right)+\lambda_{\max }\left(\Pi_{i}\right)<0, \\
\alpha\left(Q_{1}+d_{2} Q_{2}\right)-(1-\mu) Q_{2}<0, \\
\alpha d_{2}-1<0, \\
\alpha \sigma^{2}-\sigma(1-\kappa)(1-v)<0,
\end{gathered}
$$

for $i \in S$.

By the weak infinitesimal operator along (2.6), it is obtained from (3.21) and (3.22) that

$$
\begin{aligned}
e^{\alpha t} E V(t, x(t), i) & \leq E V(0, x(0), i)+\int_{0}^{t} E \mathfrak{L}\left[e^{\alpha s} V(s, x(s), i)\right] d s \\
& \leq E V(0, x(0), i) .
\end{aligned}
$$

Using the definition of the Lyapunov functional (3.4) again, we have

$$
\begin{aligned}
E V(0, x(0), i)= & E\left[x(0)-D_{i} x(-d(0))\right]^{T} P_{i}\left[x(0)-D_{i} x(-d(0))\right]+\int_{-d(0)}^{0} E x^{T}(\mathrm{~s}) Q_{1} x(s) d s \\
& +\int_{-d(0)}^{0} \int_{s}^{0} E x^{T}(\theta) Q_{2} x(\theta) d \theta d s+\int_{-d_{2}}^{-d_{1}} \int_{s}^{0} \int_{\theta}^{0} E x^{T}(u) Q_{3} x(u) d u d \theta d s \\
& +\sigma \int_{-\sigma(0)}^{0} \int_{s}^{0} x^{T}(\theta) Q_{4} x(\theta) d \theta d s \\
\leq & {\left[2 \lambda_{\max }\left(\Pi_{i}\right)+d_{2} \lambda_{\max }\left(Q_{1}\right)+\tau^{2} \lambda_{\max }\left(Q_{2}\right)+\left(d_{2}-d_{1}\right) \tau^{2} \lambda_{\max }\left(Q_{3}\right)+\sigma^{3} \lambda_{\max }\left(Q_{4}\right)\right] } \\
& \times \sup _{\theta \in[-\tau, 0]} E|\varphi(\theta)|^{2} \\
\triangleq & M_{i},
\end{aligned}
$$

for $i \in S$.

Thus, from (3.23) and (3.24), it follows that

$$
E\left|x(t)-D_{i} x(t-d(t))\right|^{2} \leq \frac{1}{\Theta} M e^{-\alpha t},
$$

where $\Theta=\min _{i \in S}\left\{\lambda_{\min }\left(P_{i}\right)\right\}$ and $M=\max _{i \in S}\left\{M_{i}\right\}$. 
From $\left|D_{i}\right|<1(i \in S)$, we obtain that there exist a positive scalar $l>0$ such that $l=\max _{i \in S}\left|D_{i}\right|<1$. So, for all $\varepsilon \in(0, \min \{\alpha,-(2 / \tau) \log |l|\})$ and any $\theta>0$, by using the elementary inequality, we derive

$$
\begin{aligned}
e^{\varepsilon t} E|x(t)|^{2} & =e^{\varepsilon t} E\left|x(t)-D_{i} x(t-d(t))+D_{i} x(t-d(t))\right|^{2} \\
& \leq(1+\theta) e^{\varepsilon t} E\left|x(t)-D_{i} x(t-d(t))\right|^{2}+\left(1+\frac{1}{\theta}\right) e^{\varepsilon t} E\left|D_{i} x(t-d(t))\right|^{2} \\
& \leq \frac{1+\theta}{\Theta} M e^{-(\alpha-\varepsilon) t}+\left(1+\frac{1}{\theta}\right) e^{\varepsilon t} E\left|D_{i} x(t-d(t))\right|^{2} \\
& \leq \frac{1+\theta}{\Theta} M+l^{2}\left(1+\frac{1}{\theta}\right) e^{\varepsilon \tau} e^{\varepsilon(t-\tau(t))} E|x(t-d(t))|^{2} .
\end{aligned}
$$

From for all $\varepsilon \in(0, \min \{\alpha,-(2 / \tau) \log |l|\})$, we have $l^{2} e^{\varepsilon \tau}<1$. Thus, we can choose $\theta$ sufficiently large such that

$$
\Delta \triangleq l^{2}\left(1+\frac{1}{\theta}\right) e^{\varepsilon \tau}<1
$$

So,

$$
e^{\varepsilon t} E|x(t)|^{2} \leq \frac{1+\theta}{\Theta} M+\Delta e^{\varepsilon(t-d(t))} E|x(t-d(t))|^{2} .
$$

For all $T>0$, from (3.28), it follows that

$$
\sup _{0 \leq t \leq T}\left[e^{\varepsilon t} E|x(t)|^{2}\right] \leq \frac{1+\theta}{\Theta} M+\Delta E|\varphi|^{2}+\Delta \sup _{0 \leq t \leq T}\left[e^{\varepsilon t} E|x(t)|^{2}\right],
$$

that is,

$$
\sup _{0 \leq t \leq T}\left[e^{\varepsilon t} E|x(t)|^{2}\right] \leq \frac{((1+\theta) / \Theta) M+\Delta E|\varphi|^{2}}{1-\Delta} .
$$

When $T \rightarrow+\infty$, it follows from (3.30) that

$$
\sup _{0 \leq t<\infty}\left[e^{\varepsilon t} E|x(t)|^{2}\right] \leq \frac{((1+\theta) / \Theta) M+\Delta E|\varphi|^{2}}{1-\Delta}
$$

So, we can obtain

$$
E|x(t)|^{2} \leq \frac{((1+\theta) / \Theta) M+\Delta E|\varphi|^{2}}{1-\Delta} e^{-\varepsilon t}
$$

The proof of this theorem is completed. 
Theorem 3.2. Suppose that Assumptions 2.1-2.4 hold and for any given positive scalar $\kappa \in(0,1)$, the uncertain neutral stochastic systems with mixed delays and Markovian switching (2.3) are robustly exponentially stable in mean square if there exist $\lambda_{i}>0(i \in S), \varepsilon_{1 i}>0, \varepsilon_{2 i}>0(i \in S)$, and some positive definite matrices $P_{i}>0(i \in S)$ and $Q_{l}>0(l=1,2, \ldots, 10)$ such that the following linear matrix inequalities (LMIs) are satisfied:

$$
P_{i}<\lambda_{i} I, \quad i \in S,
$$

$$
\Omega_{i}=\left[\begin{array}{ccccccccccc}
\Omega_{11} & \Omega_{12} & 0 & \Omega_{14} & \Omega_{15} & \Omega_{16} & \Omega_{17} & \Omega_{18} & \Omega_{19} & \Omega_{110} & 0 \\
* & \Omega_{22} & 0 & 0 & 0 & 0 & \Omega_{27} & \Omega_{28} & \Omega_{29} & 0 & \Omega_{211} \\
* & * & \Omega_{33} & 0 & 0 & 0 & 0 & 0 & 0 & 0 & 0 \\
* & * & * & \Omega_{44} & \Omega_{45} & \Omega_{46} & 0 & 0 & 0 & 0 & 0 \\
* & * & * & * & \Omega_{55} & \Omega_{56} & 0 & 0 & 0 & 0 & 0 \\
* & * & * & * & * & \Omega_{66} & 0 & 0 & 0 & 0 & 0 \\
* & * & * & * & * & * & \Omega_{77} & \Omega_{78} & \Omega_{79} & 0 & 0 \\
* & * & * & * & * & * & * & \Omega_{88} & \Omega_{89} & 0 & 0 \\
* & * & * & * & * & * & * & * & \Omega_{99} & 0 & 0 \\
* & * & * & * & * & * & * & * & * & -\varepsilon_{1 i} I & 0 \\
* & * & * & * & * & * & * & * & * & * & -\varepsilon_{2 i} I
\end{array}\right]<0, \quad i \in S,
$$

where $*$ denotes the entries that are readily inferred by symmetry of a symmetric matrix and

$$
\begin{aligned}
\Omega_{11}= & P_{i} A_{1 i}+A_{1 i}^{T} P_{i}+\sum_{j=1}^{N} \gamma_{i j} P_{j}+\lambda_{i} R_{1 i}^{T} R_{1 i}+L^{T}\left(Q_{5}+Q_{8}\right) L+Q_{1}+d_{2} Q_{2}+\frac{1}{2}\left(d_{2}^{2}-d_{1}^{2}\right) Q_{3} \\
& +\sigma^{2} Q_{4}+\left(\varepsilon_{1 i}+\varepsilon_{2 i}\right) N_{1 i}^{T} N_{1 i}, \\
\Omega_{12}= & A_{1 i}^{T} P_{i} D_{i}-\sum_{j=1}^{N} \gamma_{i j} P_{j} D_{i}, \quad \Omega_{14}=P_{i} A_{2 i}+\varepsilon_{1 i} N_{1 i}^{T} N_{2 i}, \quad \Omega_{15}=P_{i} A_{3 i}+\varepsilon_{1 i} N_{1 i}^{T} N_{3 i}, \quad \Omega_{110}=P_{i} M_{i}, \\
\Omega_{16}= & P_{i} A_{4 i}+\varepsilon_{1 i} N_{1 i}^{T} N_{4 i}, \quad \Omega_{17}=\varepsilon_{2 i} N_{1 i}^{T} N_{2 i}, \quad \Omega_{18}=\varepsilon_{2 i} N_{1 i}^{T} N_{3 i}, \quad \Omega_{19}=\varepsilon_{2 i} N_{1 i}^{T} N_{4 i}, \\
\Omega_{22}= & L^{T}\left(Q_{6}+Q_{9}\right) L+\sum_{j=1}^{N} \gamma_{i j} D_{i}^{T} P_{j} D_{i}+\lambda_{i} R_{2 i}^{T} R_{2 i}-(1-\mu) Q_{1}, \quad \Omega_{27}=D_{i}^{T} P_{i} A_{2 i}, \\
\Omega_{28}= & D_{i}^{T} P_{i} A_{3 i}, \quad \Omega_{29}=D_{i}^{T} P_{i} A_{4 i}, \quad \Omega_{211}=D_{i}^{T} P_{i} M_{i}, \\
\Omega_{33}= & L^{T}\left(Q_{7}+Q_{10}\right) L+\lambda_{i} R_{3 i}^{T} R_{3 i}-\kappa(1-v) Q_{4}, \quad \Omega_{44}=-Q_{5}+\varepsilon_{1 i} N_{2 i}^{T} N_{2 i}, \quad \Omega_{45}=\varepsilon_{1 i} N_{2 i}^{T} N_{3 i}, \\
\Omega_{46}= & \varepsilon_{1 i} N_{2 i}^{T} N_{4 i}, \quad \Omega_{55}=-Q_{6}+\varepsilon_{1 i} N_{3 i}^{T} N_{3 i}, \quad \Omega_{56}=\varepsilon_{1 i} N_{3 i}^{T} N_{4 i}, \quad \Omega_{66}=-Q_{7}+\varepsilon_{1 i} N_{4 i}^{T} N_{4 i}, \\
\Omega_{77}= & -Q_{8}+\varepsilon_{2 i} N_{2 i}^{T} N_{2 i}, \quad \Omega_{78}=\varepsilon_{2 i} N_{2 i}^{T} N_{3 i}, \quad \Omega_{79}=\varepsilon_{2 i} N_{2 i}^{T} N_{4 i}, \\
\Omega_{88}= & -Q_{9}+\varepsilon_{2 i} N_{3 i}^{T} N_{3 i}, \quad \Omega_{89}=\varepsilon_{2 i} N_{3 i}^{T} N_{4 i}, \quad \Omega_{99}=-Q_{10}+\varepsilon_{2 i} N_{4 i}^{T} N_{4 i} .
\end{aligned}
$$


Proof. Replacing $A_{1 i}, A_{2 i}, A_{3 i}$, and $A_{4 i}$ in (3.2) with $A_{1 i}+\Delta A_{1 i}(t), A_{2 i}+\Delta A_{2 i}(t), A_{3 i}+\Delta A_{3 i}(t)$, and $A_{4 i}+\Delta A_{4 i}(t), \Delta A_{1 i}(t), \Delta A_{2 i}(t), \Delta A_{3 i}(t), \Delta A_{4 i}(t)$ are described in (2.4) and (2.5), in view of Lemma 2.9 and Lemma 2.10, we obtain

$$
\begin{aligned}
\Pi_{i}(t) & =\Pi_{i}+\Xi_{i}^{T} F_{i}(t) E_{1 i}+E_{1 i}^{T} F_{i}^{T}(t) \Xi_{i}+\Psi_{i}^{T} F_{i}(t) E_{2 i}+E_{2 i}^{T} F_{i}^{T}(t) \Psi_{i} \\
& =\Pi_{i}+\varepsilon_{1 i}^{-1} \Xi_{i} \Xi_{i}^{T}+\varepsilon_{1 i} E_{1 i}^{T} E_{1 i}+\varepsilon_{2 i}^{-1} \Psi_{i} \Psi_{i}^{T}+\varepsilon_{2 i} E_{2 i}^{T} E_{2 i} \\
& =\Omega_{i}<0,
\end{aligned}
$$

where

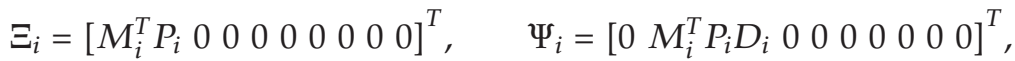

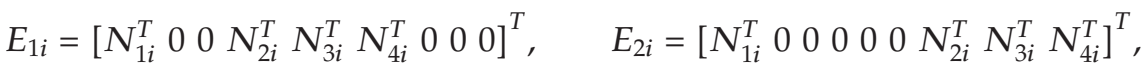

where $\varepsilon_{1 i}>0$ and $\varepsilon_{2 i}>0$, for any $i \in S$. The proof of the remainder can be easily finished by following a similar line as in the proof of Theorem 3.1. The proof is completed.

Remark 3.3. The delay-dependent sufficient conditions ensuring the robustly exponential stability in mean square of the uncertain neutral stochastic systems with mixed delays and Markovian switching (2.3) are provided in Theorem 3.2. It should be pointed out that such conditions are given in the form of LMIs, which could be easily solved by using the standard software packages. Besides, the criteria derived are dependent upon both the upper and lower bound of the time-varying delay and the distributed delay, which are less conservative.

Remark 3.4. Besides, by the Borel-Cantelli Lemma, we can also obtain the almost surely exponential stability of systems (2.3). Here, for the sake of brevity, we omit it and the readers can refer to [17]. Besides, we can easily come to a conclusion that the uncertain neutral stochastic delay systems with mixed delays and Markovian jumping parameters (2.3) are asymptotically stable in mean square from the conditions $\left|D_{i}\right|<1(i \in S)$. Thus, the results given in [15] are generalized.

Case 1. Consider the problem of delay-dependent robust exponential stability for a special case of the uncertain neutral stochastic systems with mixed delays and Markovian switching in (2.6), that is,

$$
\begin{aligned}
d\left[x(t)-D_{i} x(t-d(t))\right] & \\
= & {\left[A_{1 i}(t) x(t)+A_{2 i}(t) f(x(t))+A_{3 i}(t) f(x(t-d(t)))+A_{4 i}(t) \int_{t-\sigma(t)}^{t} f(x(s)) d s\right] d t } \\
& +\left[A_{5 i}(t) x(t)+A_{6 i}(t) x(t-d(t))+A_{7 i}(t) \int_{t-\sigma(t)}^{t} x(s) d s\right] d B(t), \quad t \geq 0,
\end{aligned}
$$


where $A_{1 i}(t), A_{2 i}(t), A_{3 i}(t)$, and $A_{4 i}(t)$ are given in (2.3), while $A_{5 i}(t), A_{6 i}(t)$, and $A_{7 i}(t)$ are of the following form:

$$
\left[A_{5 i}(t) A_{6 i}(t) A_{7 i}(t)\right]=\left[\begin{array}{lll}
A_{5 i} & A_{6 i} & A_{7 i}
\end{array}\right]+M_{i} F_{i}(t)\left[N_{5 i} N_{6 i} N_{7 i}\right]
$$

where $A_{5 i}, A_{6 i}, A_{7 i}, M_{i}, N_{5 i}, N_{6 i}$, and $N_{7 i}(i \in S)$ are known constant matrices. For systems (3.37), we can also obtain the robust exponential stability. The proof can be easily established by following a similar line as in the proof of Theorem 3.1 and then is omitted here.

Theorem 3.5. Suppose that Assumptions 2.1 and 2.2 hold and for any given positive scalar $\kappa \in$ $(0,1)$, the uncertain neutral stochastic systems with mixed delays and Markovian switching (3.37) are robustly exponentially stable in mean square if there exist $\lambda_{i}>0, \varepsilon_{1 i}>0, \varepsilon_{2 i}>0, \varepsilon_{3 i}>0$ (i $i \in S$ ) and some positive definite matrices $P_{i}>0(i \in S)$ and $Q_{i}>0(i=1,2, \ldots, 10)$ such that the following linear matrix inequalities (LMIs) are satisfied:

$$
P_{i} \leq \lambda_{i} I, \quad i \in S,
$$

$$
\left[\begin{array}{ccccccccccccc}
\Gamma_{11} & \Gamma_{12} & \Gamma_{13} & \Gamma_{14} & \Gamma_{15} & \Gamma_{16} & 0 & 0 & 0 & A_{5 i}^{T} P_{i} & P_{i} M_{i} & 0 & 0 \\
* & \Gamma_{22} & \Gamma_{23} & 0 & 0 & 0 & \Gamma_{27} & \Gamma_{28} & \Gamma_{29} & A_{6 i}^{T} P_{i} & 0 & D_{i}^{T} P_{i} M_{i} & 0 \\
* & * & \Gamma_{33} & 0 & 0 & 0 & 0 & 0 & 0 & A_{7 i}^{T} P_{i} & 0 & 0 & 0 \\
* & * & * & \Gamma_{44} & \Gamma_{45} & \Gamma_{46} & 0 & 0 & 0 & 0 & 0 & 0 & 0 \\
* & * & * & * & \Gamma_{55} & \Gamma_{56} & 0 & 0 & 0 & 0 & 0 & 0 & 0 \\
* & * & * & * & * & \Gamma_{66} & 0 & 0 & 0 & 0 & 0 & 0 & 0 \\
* & * & * & * & * & * & \Gamma_{77} & \Gamma_{78} & \Gamma_{79} & 0 & 0 & 0 & 0 \\
* & * & * & * & * & * & * & \Gamma_{88} & \Gamma_{89} & 0 & 0 & 0 & 0 \\
* & * & * & * & * & * & * & * & \Gamma_{99} & 0 & 0 & 0 & 0 \\
* & * & * & * & * & * & * & * & * & -P_{i} & 0 & 0 & P_{i} M_{i} \\
* & * & * & * & * & * & * & * & * & * & -\varepsilon_{1 i} I & 0 & 0 \\
* & * & * & * & * & * & * & * & * & * & * & -\varepsilon_{2 i} I & 0 \\
* & * & * & * & * & * & * & * & * & * & * & * & -\varepsilon_{3 i} I
\end{array}\right]<0,
$$

where $*$ denotes the entries that are readily inferred by symmetry of a symmetric matrix and

$$
\begin{aligned}
\Gamma_{11}= & P_{i} A_{1 i}+A_{1 i}^{T} P_{i}+\sum_{j=1}^{N} \gamma_{i j} P_{j}+L^{T}\left(Q_{5}+Q_{8}\right) L+Q_{1}+d_{2} Q_{2}+\frac{1}{2}\left(d_{2}^{2}-d_{1}^{2}\right) Q_{3}+\sigma^{2} Q_{5} \\
& +\varepsilon_{1 i} N_{1 i}^{T} N_{1 i}+\varepsilon_{3 i} N_{5 i}^{T} N_{5 i}, \\
\Gamma_{12}= & A_{1 i}^{T} P_{i} D_{i}-\sum_{j=1}^{N} \gamma_{i j} P_{j} D_{i}^{T}+\varepsilon_{3 i} N_{5 i}^{T} N_{6 i}, \quad \Gamma_{13}=\varepsilon_{3 i} N_{5 i}^{T} N_{7 i}, \quad \Gamma_{14}=P_{i} A_{2 i}^{T}+\varepsilon_{1 i} N_{1 i}^{T} N_{2 i}, \\
\Gamma_{15}= & P_{i} A_{3 i}+\varepsilon_{1 i} N_{1 i}^{T} N_{3 i}, \quad \Gamma_{16}=P_{i} A_{4 i}+\varepsilon_{1 i} N_{1 i}^{T} N_{4 i},
\end{aligned}
$$




$$
\begin{aligned}
& \Gamma_{22}=L^{T}\left(Q_{6}+Q_{9}\right) L+\sum_{j=1}^{N} \gamma_{i j} D_{i}^{T} P_{j} D_{i}-(1-\mu) Q_{1}+\varepsilon_{3 i} N_{6 i}^{T} N_{6 i}, \quad \Gamma_{23}=\varepsilon_{3 i} N_{6 i}^{T} N_{7 i}, \\
& \Gamma_{27}=D_{i}^{T} P_{i} A_{2 i}, \quad \Gamma_{28}=D_{i}^{T} P_{i} A_{3 i}, \quad \Gamma_{29}=D_{i}^{T} P_{i} A_{3 i}, \\
& \Gamma_{33}=L^{T}\left(Q_{7}+Q_{10}\right) L-\kappa(1-v) Q_{5}+\varepsilon_{3 i} N_{7 i}^{T} N_{7 i}, \quad \Gamma_{44}=-Q_{5}+\varepsilon_{1 i} N_{2 i}^{T} N_{2 i}, \quad \Gamma_{45}=\varepsilon_{1 i} N_{2 i}^{T} N_{3 i}, \\
& \Gamma_{46}=\varepsilon_{1 i} N_{2 i}^{T} N_{4 i}, \quad \Gamma_{55}=-Q_{6}+\varepsilon_{1 i} N_{3 i}^{T} N_{3 i}, \quad \Gamma_{56}=\varepsilon_{1 i} N_{3 i}^{T} N_{4 i}, \quad \Gamma_{66}=-Q_{7}+\varepsilon_{1 i} N_{4 i}^{T} N_{4 i}, \\
& \Gamma_{77}=-Q_{8}+\varepsilon_{2 i} N_{2 i}^{T} N_{2 i}, \quad \Gamma_{78}=\varepsilon_{2 i} N_{2 i}^{T} N_{3 i}, \quad \Gamma_{79}=\varepsilon_{2 i} N_{2 i}^{T} N_{4 i}, \quad \Gamma_{88}=-Q_{9}+\varepsilon_{2 i} N_{3 i}^{T} N_{3 i}, \\
& \Gamma_{89}=\varepsilon_{2 i} N_{3 i}^{T} N_{4 i}, \quad \Gamma_{99}=\varepsilon_{2 i} N_{4 i}^{T} N_{4 i} .
\end{aligned}
$$

Case 2. When $\sigma=0$, the uncertain neutral stochastic systems (2.3) are described as

$$
\begin{gathered}
d\left[x(t)-D_{i} x(t-d(t))\right]=\left[A_{1 i}(t) x(t)+A_{2 i}(t) f(x(t))+A_{3 i}(t) f(x(t-d(t)))\right. \\
\left.+A_{4 i}(t) \int_{t-\sigma(t)}^{t} f(x(s)) d s\right] d t, \quad t \geq 0 .
\end{gathered}
$$

Theorem 3.6. Suppose that Assumptions 2.1 and 2.2 hold and for any given positive scalar $\kappa \in$ $(0,1)$, the uncertain neutral stochastic systems with mixed delays and Markovian switching (3.41) are robustly exponentially stable in mean square if there exist $\lambda_{i}>0(i \in S), \varepsilon_{i}>0(i \in S)$ and some positive definite matrices $P_{i}>0(i \in S)$ and $Q_{i}>0(i=1,2, \ldots, 10)$ such that the following linear matrix inequalities (LMIs) are satisfied: for $i \in S$,

$$
\Omega_{i}=\left[\begin{array}{ccccccccccc}
\Omega_{11} & \Omega_{12} & 0 & \Omega_{14} & \Omega_{15} & \Omega_{16} & \Omega_{17} & \Omega_{18} & \Omega_{19} & \Omega_{110} & 0 \\
* & \Omega_{22} & 0 & 0 & 0 & 0 & \Omega_{27} & \Omega_{28} & \Omega_{29} & 0 & \Omega_{211} \\
* & * & \Omega_{33} & 0 & 0 & 0 & 0 & 0 & 0 & 0 & 0 \\
* & * & * & \Omega_{44} & \Omega_{45} & \Omega_{46} & 0 & 0 & 0 & 0 & 0 \\
* & * & * & * & \Omega_{55} & \Omega_{56} & 0 & 0 & 0 & 0 & 0 \\
* & * & * & * & * & \Omega_{66} & 0 & 0 & 0 & 0 & 0 \\
* & * & * & * & * & * & \Omega_{77} & \Omega_{78} & \Omega_{79} & 0 & 0 \\
* & * & * & * & * & * & * & \Omega_{88} & \Omega_{89} & 0 & 0 \\
* & * & * & * & * & * & * & * & \Omega_{99} & 0 & 0 \\
* & * & * & * & * & * & * & * & * & -\varepsilon_{1 i} I & 0 \\
* & * & * & * & * & * & * & * & * & * & -\varepsilon_{2 i} I
\end{array}\right]<0, \quad i \in S,
$$


where $*$ denotes the entries that are readily inferred by symmetry of a symmetric matrix and

$$
\begin{aligned}
\Omega_{11}= & P_{i} A_{1 i}+A_{1 i}^{T} P_{i}+\sum_{j=1}^{N} \gamma_{i j} P_{j}+L^{T}\left(Q_{5}+Q_{8}\right) L+Q_{1}+d_{2} Q_{2}+\frac{1}{2}\left(d_{2}^{2}-d_{1}^{2}\right) Q_{3}+\sigma^{2} Q_{4} \\
& +\left(\varepsilon_{1 i}+\varepsilon_{2 i}\right) N_{1 i}^{T} N_{1 i}, \\
\Omega_{22}= & L^{T}\left(Q_{6}+Q_{9}\right) L+\sum_{j=1}^{N} \gamma_{i j} D_{i}^{T} P_{j} D_{i}-(1-\mu) Q_{1}, \quad \Omega_{27}=D_{i}^{T} P_{i} A_{2 i}, \\
\Omega_{33}= & L^{T}\left(Q_{7}+Q_{10}\right) L-\kappa(1-v) Q_{4},
\end{aligned}
$$

and $\Omega_{12}, \Omega_{14}, \Omega_{15}, \Omega_{16}, \Omega_{17}, \Omega_{18}, \Omega_{19}, \Omega_{27}, \Omega_{28}, \Omega_{29}, \Omega_{33}, \Omega_{44}, \Omega_{45}, \Omega_{46}, \Omega_{55}, \Omega_{56}, \Omega_{66}, \Omega_{77}, \Omega_{78}$, $\Omega_{79}, \Omega_{88}, \Omega_{89}$, and $\Omega_{99}$ are given in Theorem 3.2.

Remark 3.7. The sufficient conditions ensuring the exponential stability in mean square of the uncertain neutral delay systems with Markovian jumping parameters in [39,40] are dependent upon the upper bound of the exponential stability rate. But, Theorem 3.6 can remove this restrictive conditions. So, we can improve and generalize the results in $[39,40]$.

\section{Numerical Examples}

In this section, two examples are provided to illustrate the feasibility and applicability of our obtained results.

Example 4.1. Consider the case of 2D Brownian motion, and $r(t)$ is right-continuous Markovian chain taking values in $S=\{1,2\}$ with its generator $\Gamma=\left[\begin{array}{cc}-3 & 3 \\ 4 & -4\end{array}\right]$. And the parameters in systems (2.3) are given as follows:

$$
\begin{gathered}
A_{11}=\left[\begin{array}{cc}
-1.5 & 0 \\
0 & -1.8
\end{array}\right], \quad A_{12}=\left[\begin{array}{cc}
-1.8 & 0 \\
0 & -1.8
\end{array}\right], \quad A_{21}=\left[\begin{array}{cc}
0.3 & 0.2 \\
-0.6 & -0.8
\end{array}\right], \quad A_{22}=\left[\begin{array}{cc}
0.6 & -0.5 \\
-0.4 & -0.3
\end{array}\right], \\
A_{31}=\left[\begin{array}{cc}
-0.7 & -0.5 \\
0.4 & 0.6
\end{array}\right], \quad A_{32}=\left[\begin{array}{cc}
0.7 & 0.5 \\
-0.3 & 0.3
\end{array}\right], \quad A_{41}=\left[\begin{array}{cc}
0.2 & 0.3 \\
-0.1 & 0.2
\end{array}\right], \quad A_{42}=\left[\begin{array}{cc}
0.3 & 0.2 \\
-0.2 & 0.4
\end{array}\right], \\
D_{1}=\left[\begin{array}{cc}
-0.2 & 0.0 \\
1.0 & 0.1
\end{array}\right], \quad D_{2}=\left[\begin{array}{ll}
0.1 & 0.0 \\
0.7 & 0.2
\end{array}\right], \quad M_{1}=\left[\begin{array}{ll}
0.1 & 0.2 \\
0.0 & 0.1
\end{array}\right], \quad M_{2}=\left[\begin{array}{ll}
0.2 & 0.1 \\
0.1 & 0.2
\end{array}\right], \\
N_{11}=N_{12}=N_{21}=N_{22}=N_{31}=N_{32}=N_{41}=N_{42}=\left[\begin{array}{ll}
0.1 & 0.1
\end{array}\right] .
\end{gathered}
$$

The delays $d(t)=0.45 \sin (t)+0.3, \sigma(t)=0.4 \cos (t)+0.4$, and it is easily obtained that $d_{1}=0.15$, $d_{2}=0.75, \mu=0.45, v=0.4$, and $\sigma=0.8$. Assume that the activation function $f$ satisfies 
Assumption 2.1 with the Lipschitz coefficient matrix $L=\left[\begin{array}{ll}0.2 & 0.0 \\ 0.0 & 0.2\end{array}\right]$ and the function $\sigma$ satisfies Assumption 2.2 with the matrices $R_{i j}(i=1,2 ; j=1,2,3)$ given by

$$
\begin{array}{cc}
R_{11}=\left[\begin{array}{cc}
0.15 & 0.0 \\
0.20 & 0.35
\end{array}\right], \quad R_{12}=\left[\begin{array}{cc}
0.5 & 0.1 \\
0.28 & 0.20
\end{array}\right], \quad R_{21}=\left[\begin{array}{ll}
0.3 & 0.0 \\
0.2 & 0.1
\end{array}\right], \quad R_{22}=\left[\begin{array}{ll}
0.1 & 0.0 \\
0.2 & 0.1
\end{array}\right], \\
R_{31}=\left[\begin{array}{ll}
0.1 & 0.2 \\
0.1 & 0.2
\end{array}\right], \quad R_{32}=\left[\begin{array}{ll}
0.1 & 0.1 \\
0.2 & 0.1
\end{array}\right] .
\end{array}
$$

With the parameters above, when $\kappa=0.9999$, by using Matlab LMI Toolbox, according to Theorem 3.2, we solve LMIs (3.33) and obtain there feasible solutions as follows:

$$
\begin{aligned}
& P_{1}=\left[\begin{array}{ll}
70.0930 & 12.2285 \\
12.2285 & 21.8248
\end{array}\right], \quad P_{2}=\left[\begin{array}{ll}
74.1549 & 15.4873 \\
15.4873 & 20.7284
\end{array}\right], \quad Q_{1}=\left[\begin{array}{cc}
134.4662 & 25.4223 \\
25.4223 & 20.5296
\end{array}\right], \\
& Q_{2}=\left[\begin{array}{cc}
1.8693 & -0.0065 \\
-0.0065 & 0.6213
\end{array}\right], \quad Q_{3}=\left[\begin{array}{cc}
4.8828 & -0.0153 \\
-0.0153 & 1.6598
\end{array}\right], \quad Q_{4}=\left[\begin{array}{cc}
16.7672 & -0.0153 \\
-0.0153 & 1.6598
\end{array}\right] \text {, } \\
& Q_{5}=\left[\begin{array}{rr}
117.1703 & -17.8696 \\
-17.8696 & 119.1560
\end{array}\right], \quad Q_{6}=\left[\begin{array}{rr}
114.7061 & 67.0438 \\
67.0438 & 91.0274
\end{array}\right], \quad Q_{7}=\left[\begin{array}{ll}
58.3284 & 23.0811 \\
23.0811 & 70.1310
\end{array}\right] \text {, } \\
& Q_{8}=\left[\begin{array}{ll}
57.0181 & 12.7280 \\
12.7280 & 58.0073
\end{array}\right], \quad Q_{9}=\left[\begin{array}{ll}
41.6921 & 14.6919 \\
14.6919 & 40.8478
\end{array}\right], \quad Q_{10}=\left[\begin{array}{ll}
40.5029 & -0.2295 \\
-0.2295 & 33.2920
\end{array}\right] \text {, } \\
& \lambda_{1}=75.3750, \quad \lambda_{2}=80.6197, \quad \varepsilon_{11}=66.1002, \quad \varepsilon_{12}=97.9532, \quad \varepsilon_{21}=47.7433, \quad \varepsilon_{22}=56.9671 \text {. }
\end{aligned}
$$

Example 4.2. Let $r(t)$ be right-continuous Markovian chain taking values in $S=\{1,2\}$ with its generator $\Gamma=\left[\begin{array}{cc}-2 & 2 \\ 1 & -1\end{array}\right]$. Consider the following uncertain neutral systems with mixed delays and Markovian switching: for any $i \in S$,

$$
\frac{d}{d t}\left[x(t)-D_{i} x(t-d(t))\right]=A_{1 i}(t) x(t)+A_{2 i}(t) x(t-d(t)), \quad t \geq 0,
$$

where

$$
\begin{gathered}
A_{11}=A_{12}=\left[\begin{array}{ll}
-2 & 0.0 \\
0.0 & -3
\end{array}\right], \quad A_{21}=A_{22}=\left[\begin{array}{cc}
-1 & 0 \\
-1 & -1
\end{array}\right], \quad D_{1}=\left[\begin{array}{cc}
0.3 & 0 \\
0 & 0.3
\end{array}\right], \\
D_{2}=\left[\begin{array}{ll}
0.1 & 0.0 \\
0.0 & 0.1
\end{array}\right], \quad M_{1}=\left[\begin{array}{ll}
0.1 & 0.1 \\
0.2 & 0.1
\end{array}\right], \quad M_{2}=\left[\begin{array}{cc}
0.2 & -0.1 \\
0.1 & 0.1
\end{array}\right], \\
N_{11}=\left[\begin{array}{ll}
0.1 & 0.0
\end{array}\right], \quad N_{12}=\left[\begin{array}{ll}
0.0 & 0.1
\end{array}\right], \quad N_{21}=\left[\begin{array}{ll}
-0.0 & 0.1
\end{array}\right], \quad N_{22}=\left[\begin{array}{ll}
0.1 & 0.1
\end{array}\right] .
\end{gathered}
$$


Applying Matlab toolbox, by Theorem 3.6, for $\mu=0$, it can be obtained that the uncertain neutral systems with delays and Markovian jumping parameters (4.4) are robustly exponentially stable in mean square with the delay $d(t)$ satisfying $0 \leq d(t) \leq 6.0 \times 10^{17}$.

\section{Conclusions}

In this paper, the exponential stability in mean square of the uncertain neutral stochastic systems with mixed delays and Markovian jumping parameters has been considered. The mixed delays consist of the discrete delay and the distributed delay. The LMI-based conditions ensuring the exponential stability in mean square of such systems are obtained by constructing an appropriate Lyapunov functional, which are dependent upon the upper bound and lower bound of the discrete time delays and distributed delays. It is worth pointing out that, compared with the previous works $[17,18]$, the stability criteria in this paper can be easily checked by using some standard numerical packages. Two illustrative examples are provided to show the effectiveness and applicability of the proposed results.

\section{Acknowledgments}

The author would like to thank the anonymous referees for their very helpful comments and suggestions that greatly improved this paper and the editors for their careful reading of this paper. This work is supported by the National Natural Science Foundation of China under Grant no. 11126278 and Grant no. 11101202, the Natural Science Foundation of Jiangxi Province under Grant no. 20114BAB211001 and Grant no. 2009GQS0018, and the Youth Foundation of Jiangxi Provincial Educations of China under Grant no. GJJ11045 and Grant no. GJJ10051.

\section{References}

[1] S. E. A. Mohammed, Stochastic Functional Differential Equations, vol. 99 of Research Notes in Mathematics, Pitman, Boston, Mass, USA, 1984.

[2] J. Zhu, Q. Zhang, and C. Yang, “Delay-dependent robust stability for Hopfield neural networks of neutral-type," Neurocomputing, vol. 72, no. 10-12, pp. 2609-2617, 2009.

[3] W.-H. Chen and W. X. Zheng, "Delay-dependent robust stabilization for uncertain neutral systems with distributed delays," Automatica, vol. 43, no. 1, pp. 95-104, 2007.

[4] M. Wu, Y. He, and J.-H. She, "New delay-dependent stability criteria andstabilizing method for neutral systems," IEEE Transactions on Automatic Control, vol. 49, no. 12, pp. 2266-2271, 2004.

[5] Y. He, M. Wu, J.-H. She, and G.-P. Liu, "Delay-dependent robust stability criteria for uncertain neutral systems with mixed delays," Systems \& Control Letters, vol. 51, no. 1, pp. 57-65, 2004.

[6] P. Balasubramaniam and R. Rakkiyappan, "Global exponential stability for neutral-type BAM neural networks with time-varying delays," International Journal of Computer Mathematics, vol. 87, no. 9, pp. 2064-2075, 2010.

[7] R. Rakkiyappan, P. Balasubramaniam, and J. Cao, “Global exponential stability results for neutraltype impulsive neural networks," Nonlinear Analysis: Real World Applications, vol. 11, no. 1, pp. 122130, 2010.

[8] X. Mao, Stochastic Differential Equations and Applications, HorwoodPublication, Chichester, UK, 1997.

[9] L. Huang and X. Mao, "Delay-dependent exponential stability of neutral stochastic delay systems," IEEE Transactions on Automatic Control, vol. 54, no. 1, pp. 147-152, 2009.

[10] X. Mao, "Razumikhin-type theorems on exponential stability of neutral stochastic functionaldifferential equations," SIAM Journal on Mathematical Analysis, vol. 28, no. 2, pp. 389-401, 1997.

[11] H. Chen, Y. Zhang, and P. Hu, "Novel delay-dependent robust stability criteria for neutral stochastic delayed neural networks," Neurocomputing, vol. 73, no. 13-15, pp. 2554-2561, 2010. 
[12] J. Wang, Y. Chen, and W. X. Zheng, "Mean-square exponential stability analysis for stochastic systems of neutral-type," in Proceedings of the 8th World Congress on Intelligent Control and Automation (WCICA '10), pp. 3542-3547, 2010.

[13] X. Mao, "Exponential stability of stochastic delay interval systems with Markovian switching," IEEE Transactions on Automatic Control, vol. 47, no. 10, pp. 1604-1612, 2002.

[14] J. Luo, "Fixed points and stability of neutral stochastic delay differential equations," Journal of Mathematical Analysis and Applications, vol. 334, no. 1, pp. 431-440, 2007.

[15] W. Su and Y. Chen, "Global asymptotic stability analysis for neutral stochastic neural networks with time-varying delays," Communications in Nonlinear Science and Numerical Simulation, vol. 14, no. 4, pp. 1576-1581, 2009.

[16] L. Zhou and G. Hu, "Almost sure exponential stability of neutral stochastic delayed cellular neural networks," Journal of Control Theory and Applications, vol. 6, no. 2, pp. 195-200, 2008.

[17] V. Kolmanovskii, N. Koroleva, T. Maizenberg, X. Mao, and A. Matasov, “Neutral stochastic differential delay equations with Markovian switching," Stochastic Analysis and Applications, vol. 21, no. 4, pp. 819-847, 2003.

[18] X. Mao, Y. Shen, and C. Yuan, "Almost surely asymptotic stability of neutral stochastic differential delay equations with Markovian switching," Stochastic Processes and their Applications, vol. 118, no. 8, pp. 1385-1406, 2008.

[19] G. Chen and Y. Shen, "Robust $H_{\infty}$ filter design for neutral stochastic uncertain systems with timevarying delay," Journal of Mathematical Analysis and Applications, vol. 353, no. 1, pp. 196-204, 2009.

[20] S. Xu, P. Shi, Y. Chu, and Y. Zou, "Robust stochastic stabilization and $H_{\infty}$ control of uncertain neutral stochastic time-delay systems," Journal of Mathematical Analysis and Applications, vol. 314, no. 1, pp. 1-16, 2006.

[21] N. N. Krasovskiı̌ and È. A. Lidskiı̌, "Analytic design of controllers in systems with random attributes. II. Equations of optimum solutions. Approximate solutions," Automation and Remote Control, vol. 22, no. 10, pp. 1141-1146, 1961.

[22] M. Mariton, Jump Linear Control Systems, Marcel Dekker, New York, NY, USA, 1990.

[23] Z. Wang, Y. Liu, L. Yu, and X. Liu, "Exponential stability of delayed recurrent neural networks with Markovian jumping parameters," Physics Letters A, vol. 356, pp. 346-352, 2006.

[24] Y. Liu, Z. Wang, and X. Liu, "On delay-dependent robust exponential stability of stochastic neural networks with mixed time delays and Markovian switching," Nonlinear Dynamics, vol. 54, no. 3, pp. 199-212, 2008.

[25] G. Wang, J. Cao, and J. Liang, "Exponential stability in the mean square for stochastic neural networks with mixed time-delays and Markovian jumping parameters," Nonlinear Dynamics, vol. 57, no. 1-2, pp. 209-218, 2009.

[26] Q. Zhu and J. Cao, "Exponential stability of stochastic neural networks with both markovian jump parameters and mixed time delays," IEEE Transactions on Systems, Man, and Cybernetics B, vol. 99, pp. $1-13,2010$.

[27] Q. Zhu and J. Cao, "Robust exponential stability of Markovian jump impulsive stochastic CohenGrossberg neural networks with mixed time delays," IEEE Transactions on Neural Networks, vol. 8, pp. 1314-1325, 2010.

[28] D. Yue and Q.-L. Han, "Delay-dependent exponential stability of stochastic systems with timevarying delay, nonlinearity, and Markovian switching," IEEE Transactions on Automatic Control, vol. 50, no. 2, pp. 217-222, 2005.

[29] X. Lou and B. Cui, "Stochastic exponential stability for Markovian jumping BAM neural networks with time-varying delays," IEEE Transactions on Systems, Man, and Cybernetics B, vol. 3, pp. 713-719, 2007.

[30] H. Zhang and Y. Wang, "Stability analysis of Markovian jumping stochastic Cohen-Grossberg neural networks with mixed time delays," IEEE Transactions on Neural Networks, vol. 2, pp. 366-370, 2008.

[31] Y. Shen and J. Wang, "Almost surely exponential stability of recurrent neural networks with Markovian switching," IEEE Transactions on Neural Networks, vol. 5, pp. 840-855, 2009.

[32] Y. Liu, Z. Wang, J. Liang, and X. Liu, "Stability and synchronization of discrete-time Markovian jumping neural networks with mixed mode-dependent time delays," IEEE Transactions on Neural Networks, vol. 7, pp. 1102-1116, 2009.

[33] S. Ma and E. K. Boukas, "Stability and robust stabilisation for uncertain discrete stochastic hybrid singular systems with time delay," IET Control Theory \& Applications, vol. 3, no. 9, pp. 1217-1225, 2009. 
[34] S. Xu, J. Lam, and X. Mao, "Delay-dependent $H_{\infty}$ control and filtering for uncertain Markovian jump systems with time-varying delays," IEEE Transactions on Circuits and Systems, vol. 54, no. 9, pp. 20702077, 2007.

[35] L. Huang and X. Mao, "On almost sure stability of hybrid stochastic systems with mode-dependent interval delays," IEEE Transactions on Automatic Control, vol. 55, no. 8, pp. 1946-1952, 2010.

[36] L. Xie, "Stochastic robust stability analysis for Markovian jumping neural networks with time delays," in Proceedings of the IEEE Networking, Sensing and Control (ICNSC '05), pp. 923-928, 2005.

[37] S. Cong, H.-T. Zhang, and Y. Zou, "A new exponential stability condition for delayed systems with Markovian switching," Acta Automatica Sinica, vol. 36, no. 7, pp. 1025-1028, 2010.

[38] C. Yuan and X. Mao, "Robust stability and controllability of stochastic differential delay equations with Markovian switching," Automatica, vol. 40, no. 3, pp. 343-354, 2004.

[39] S. He and F. Liu, "Exponential stability for uncertain neutral systems with Markov jumps," Journal of Control Theory and Applications, vol. 7, no. 1, pp. 35-40, 2009.

[40] P. Balasubramaniam, A. Manivannan, and R. Rakkiyappan, "Exponential stability results for uncertain neutral systems with interval time-varying delays and Markovian jumping parameters," Applied Mathematics and Computation, vol. 216, no. 11, pp. 3396-3407, 2010.

[41] X. Mao and C. Yuan, Stochastic Differential Equations with Markovian Switching, Imperial College Press, London, UK, 2006.

[42] S. Boyd, L. El Ghaoui, E. Feron, and V. Balakrishnan, Linear Matrix Inequalities in System and Control Theory, vol. 15 of SIAM Studies in Applied Mathematics, Society for Industrial and Applied Mathematics (SIAM), Philadelphia, Pa, USA, 1994.

[43] K. Gu, "An integral inequality in the stability problem of time-delay systems," in Proceedings of the 39th IEEE Confernce on Decision and Control, Sysdney, NSW, vol. 3, pp. 2805-2810, Sydney NSW, Australia, 2000. 


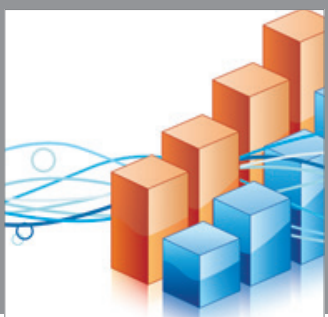

Advances in

Operations Research

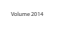

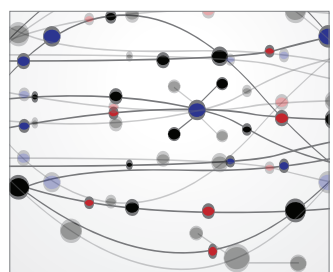

\section{The Scientific} World Journal
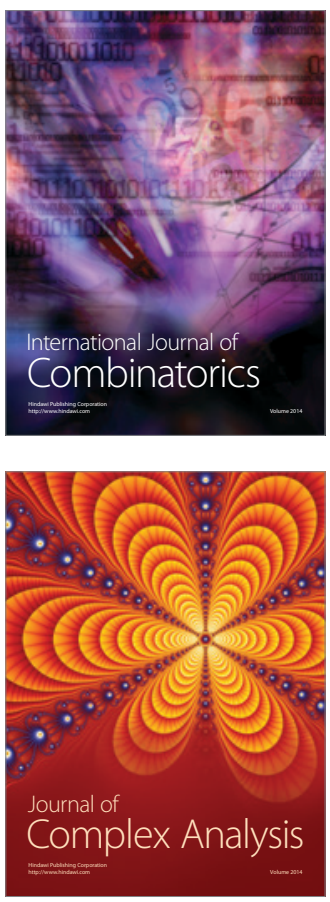

International Journal of

Mathematics and

Mathematical

Sciences
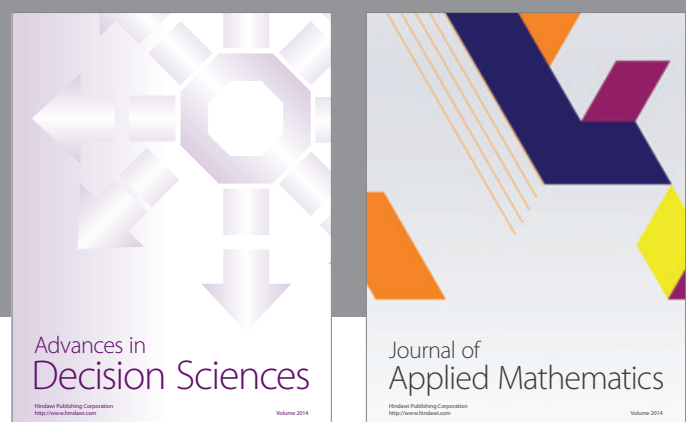

Journal of

Applied Mathematics
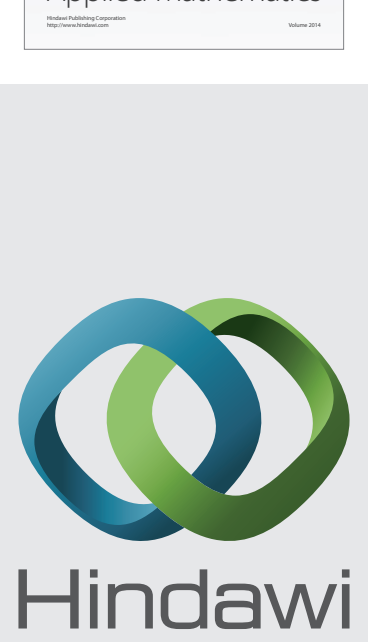

Submit your manuscripts at http://www.hindawi.com
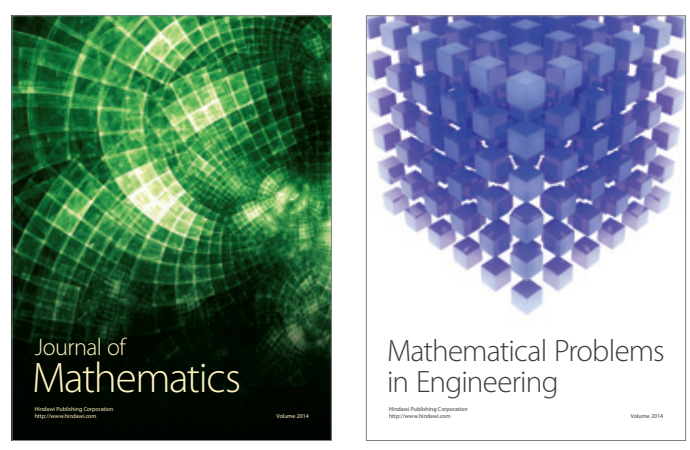

Mathematical Problems in Engineering
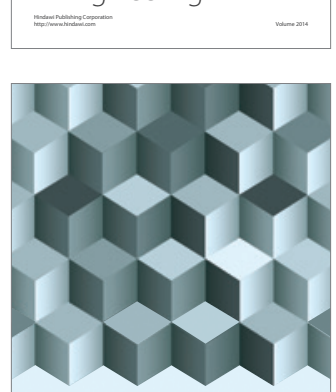

Journal of

Function Spaces
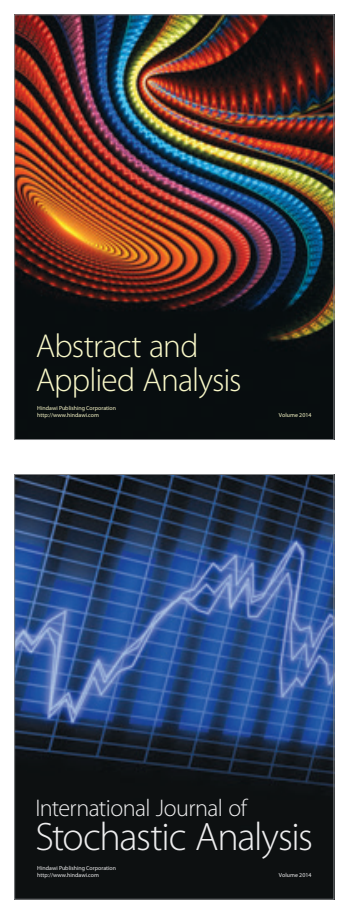

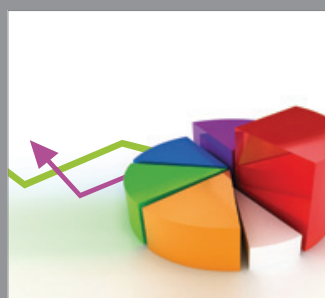

ournal of

Probability and Statistics

Promensencen
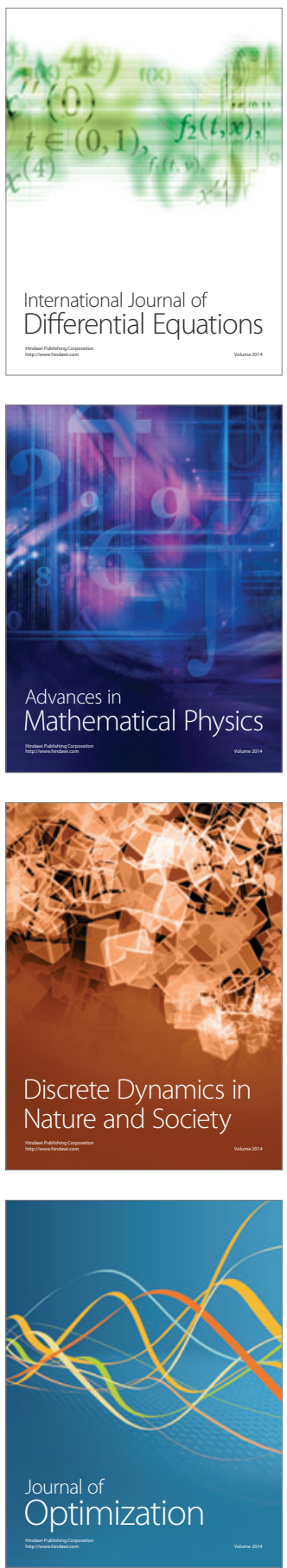